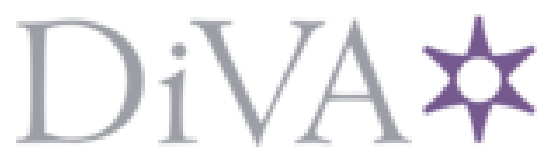

http://www.diva-portal.org

This is the published version of a paper presented at 31st European Safety and Reliability Conference (ESREL 2O21).

Citation for the original published paper:

Allahvirdizadeh, R., Karoumi, R., Andersson, A. (2021)

Surrogate-assisted versus subset simulation-based stochastic comparison between running safety and passenger comfort design criteria of high-speed railway bridges In: Bruno Castanier, Marko Cepin, David Bigaud, and Christophe Berenguer (ed.), (pp. 3334-3341).

https://doi.org/10.3850/978-981-18-2016-8 577-cd

N.B. When citing this work, cite the original published paper.

Permanent link to this version:

http://urn.kb.se/resolve?urn=urn:nbn:se:kth:diva-301310 


\section{Surrogate-assisted versus subset simulation-based stochastic comparison between running safety and passenger comfort design criteria of high-speed railway bridges}

\section{Reza Allahvirdizadeh}

Division of Structural Engineering and Bridges, KTH Royal Institute of Technology, Stockholm, Sweden. E-mail: reza.allahvirdizadeh@byv.kth.se

\section{Andreas Andersson}

Division of Structural Engineering and Bridges, KTH Royal Institute of Technology, Stockholm, Sweden. E-mail: andreas.andersson@byv.kth.se

\section{Raid Karoumi}

Division of Structural Engineering and Bridges, KTH Royal Institute of Technology, Stockholm, Sweden. E-mail: raid.karoumi@byv.kth.se

Limiting the maximum vertical acceleration and deflection of the deck are two principal design criteria of high-speed railway bridges. The former prevents ballast instability to ensure running safety, and the latter attempts to limit the acceleration of the car-body below the level at which passenger comfort is disturbed. The previous studies are mainly concerned with the destabilization of the ballast, nevertheless the possibility of the maximum deflection occurrence should not be underestimated. Moreover, the literature indicates the need to improve the current design requirements including the minimum allowable mass and frequency of bridges, which requires solving optimization problems based on modern requirements. Therefore, a probabilistic framework with simulation-based techniques is used to evaluate the violation probability of the above limit states and distinguish dominant criteria under different conditions, i.e., bridge span length and operational train speed. First, the performance of the subset simulation method is compared with the Latin Hypercube-sampling based Monte-Carlo approach supported by surrogate models. Polynomial chaos expansion (PCE) surrogate models are trained for this objective. Then, the resulting violation probabilities are evaluated for the two considered limit states using the approach with better performance.

Keywords: High-Speed Railway Bridges, Bridge Dynamics, Running Safety, Passenger Comfort, Structural Reliability, Subset Simulation, Surrogate Model, Polynomial Chaos Expansion.

\section{Introduction}

One of the most important criteria in the design of high-speed railway bridges is to ensure running safety, which can be achieved by preventing derailments. Such a limit-state can be caused by loss of contact between wheels and rail or by a phenomenon that disturbs the load path. One of these conditions is referred as ballast instability in the literature. From a theoretical point of view, ballast destabilization initiates when the induced inertial forces on the particles due to the vertical vibration of the bridge exceed the interlocking or frictional resistant forces. Therefore, CEN (2005) implicitly controls this by limiting the vertical acceleration of the deck. This approach applies a partial safety factor of 2.0 to the shaking table results obtained by Zacher and Baeßler
(2008). They recorded a significant increase in lateral displacements of the sleepers at vertical accelerations beyond $0.7 \mathrm{~g}$; which they inferred to be indicative of incipient ballast instability.

Arvidsson (2018) investigated the relationship between wheel-rail contact loss and ballast instability by performing extensive parametric studies on numerical models with traintrack-bridge interaction. She found that almost always the ballast instability is dominant. Therefore, this study formulates the violation of running safety by exceeding the vertical acceleration from the specified threshold.

However, in this context, the mentioned partial safety factor does not correspond to any specific target safety (reliability) level. In other words, it is proposed only on the basis of engineering judgments. Considering this 
shortcoming along with the necessity of satisfying sustainability requirements in designing infrastructures led Allahvirdizadeh et al. (2020) to investigate potential improvements in this subject. To achieve this goal, several approaches can be taken; one of them is to eliminate the partial safety factor and propose minimum mass and stiffness criteria instead. In this regard, Allahvirdizadeh et al. (2021) conducted Reliability-Based Design Optimization (RBDO) on simply-supported bridges with short to medium span length. The main concern that should be addressed in this approach is whether the proposed minimum allowable values conflict with other thresholds or not. This requires solving the multiobjective RBDO problem, but this would involve a large computational cost. Therefore, a prior stochastic comparison is performed in this study to distinguish circumstances under which such a conflict may occur.

The other limit state which running safety is compared is passenger comfort. The latter is assumed to be related to the car-body acceleration signal in all directions; however, the complexity of pursuing such an approach led to propose implicit control methods. In this regard, CEN (2005) indirectly verifies the passenger comfort at each train speed by limiting the relative vertical deflection of the bridge along the centerline of the track $(\delta / L)$. In this study, the performance function is formulated considering a very good comfort level. This corresponds to a vertical acceleration of 1.0 $\mathrm{m} / \mathrm{s}^{2}$ inside the coach.

In order to compare two discussed limit states, the violation probabilities of the performance functions are evaluated using simulation-based approaches. These probabilities are calculated for operational train speeds in the range of [200-300] km/h. Furthermore, the procedure is repeated for bridges with span lengths in the range of $[10.0-30.0] \mathrm{m}$.

It is worth noting that the discussed limit states correspond to events with small failure probabilities of about $10^{-3}-10^{-4}$. Therefore, it is not practical choice to use the conventional crude Monte-Carlo method as it involves significant computational costs. Therefore, the two methods of subset simulation and replacing computational models with surrogate models (also known as meta-models) are applied to speed up the evaluation procedure.

$\mathrm{Au}$ and Beck (2001) proposed the subset simulation to evaluate small failure probabilities as the product of conditional failure probabilities. They correspond to the intermediate limit states formulated in Eq.(1), which lead to larger violation probabilities and can be calculated by a smaller number of simulations. Then, the violation probability would be obtained as Eq.(2). It should also be emphasized that the conditional probabilities are estimated using the Markov chain MonteCarlo simulation technique. In this approach, the conditional probabilities are fixed at a constant value of $p_{0}$ and intermediate limit states are adapted based on this. Therefore, the performance of the subset simulation depends only on $p_{0}$ and the number of samples per iteration $\left(N_{s}\right)$.

$$
g_{i}=R-S_{i} \quad S_{1}<S_{2}<\ldots<S_{m}=S
$$

Where, $g_{i}$ is the intermediate performance function, $R$ is the capacity and $S_{i}$ is the $i$ th sequential failure event.

$$
p_{f}=P\left(g_{1}<0\right) \prod_{i=1}^{m-1} P\left(g_{i+1}<0 \mid g_{i}<0\right)
$$

Moreover, several surrogate models have been proposed to be used in reliability assessment, namely Kriging (Gaussian process regression), polynomial chaos expansion (PCE), artificial neural network (ANN), and support vector machines (SVM); where PCE is adopted here. In general, a surrogate model approximates a computational model (e.g., a finite element model) with finite variance by a function. Given a random input vector of $\mathbf{X}$ with dimension $M, \mathrm{PCE}$ would be formulated as Eq.(3). Detailed information on the concept of PCE can be found in Xiu and Karniadakis (2002); Sudret (2008).

$$
Y=\mathcal{M}(\mathbf{X}) \approx \sum_{|\alpha| \leq q} \beta_{\alpha} \psi_{\alpha}(\mathbf{X})
$$

Where, $\beta_{\alpha}$ are unknown deterministic coefficients and $\psi_{\alpha}$ are multivariate basis function; which are orthogonal with respect to joint probability distribution of basic random vector $\left(f_{\mathbf{X}}(x)\right)$. Furthermore, $\psi_{\alpha}=\prod_{i=1}^{M} \pi_{\alpha_{i}}^{(i)}\left(x_{i}\right)$; where, $\pi_{\alpha_{i}}^{(i)}$ is a family of orthogonal univariate polynomials with respect to $f_{X_{i}}$. The orthogonality is defined as Eq.(4). It is noteworthy that those families are predefined in the literature for well-known probability distributions. $|\alpha|=\sum_{i=1}^{M} \alpha_{i}$ is the degree of PCE which is less than (truncated) a given value of $q$. 


$$
\begin{array}{r}
<\pi_{k}^{(i)}\left(x_{i}\right), \pi_{l}^{(i)}\left(x_{i}\right)>=\mathbb{E}\left[\pi_{k}^{(i)}\left(x_{i}\right) \pi_{l}^{(i)}\left(x_{i}\right)\right]= \\
\int_{D_{X_{i}}} \pi_{k}^{(i)}\left(x_{i}\right) \pi_{l}^{(i)}\left(x_{i}\right) f_{X_{i}}\left(x_{i}\right) d x_{i}=\delta_{k, l}
\end{array}
$$

Where, $\delta_{k, l}$ is Kronecker delta.

\section{Computational Model}

In this study, the dynamic behavior of railway bridges is modeled by simply-supported 2D Euler-Bernoulli beams under the passage of two series of equidistant loads (representing articulated trains) moving at constant speed of $v$ (see Fig. 1). The governing differential equations are solved using the analytical solution provided by Frýba (2001). This solution is formulated as Eqs. (5)-(10).

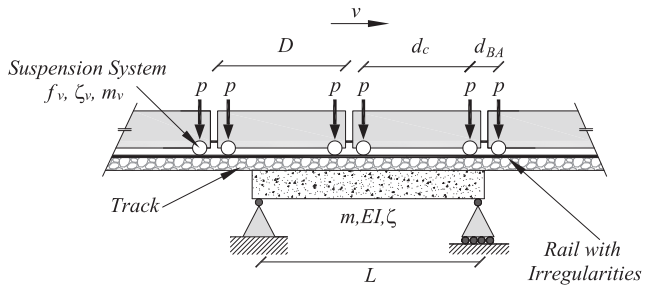

Fig. 1. Considered computational model

$$
\begin{array}{r}
y(x, t)=\sum_{i=1}^{\infty} \sum_{n=1}^{N}\left(\frac{2 p L^{3}}{\pi^{4} E I}\right) i \omega \omega_{1}^{2}\left[f_{u / a}\left(t-t_{n}\right)\right. \\
\left.\mathrm{H}\left(t-t_{n}\right)-(-1)^{i} f_{u / a}\left(t-T_{n}\right) \mathrm{H}\left(t-T_{n}\right)\right] \\
\sin \left(\frac{i \pi x}{L}\right) \\
f_{u}(t)=\frac{1}{\omega_{i}^{\prime} D}\left[\frac{\omega_{i}^{\prime}}{i \omega} \sin (i \omega t+\theta)+e^{-\omega_{d} t}\right. \\
\left.\sin \left(\omega_{i}^{\prime} t+\phi\right)\right]
\end{array}
$$

$f_{a}(t)=-\frac{\omega_{i}^{\prime 2}-\omega_{d}^{2}}{\omega_{i}^{\prime} D}\left[\frac{i \omega \omega_{i}^{\prime}}{\omega_{i}^{\prime 2}-\omega_{d}^{2}} \sin (i \omega t+\theta)+\right.$

$$
\left.e^{-\omega_{d} t} \sin \left(\omega_{i}^{\prime} t+\phi^{\prime}\right)\right]
$$

$$
\begin{gathered}
\theta=\arctan \left[\frac{-2 i \omega \omega_{d}}{\Omega_{i}^{2}-(i \omega)^{2}}\right] \\
\phi=\arctan \left[\frac{2 \omega_{d} \omega_{i}^{\prime}}{\omega_{d}^{2}-\omega_{i}^{\prime 2}+(i \omega)^{2}}\right]
\end{gathered}
$$

$$
\phi^{\prime}=\phi+\arctan \left[\frac{2 \omega_{d} \omega_{i}^{\prime}}{\omega_{i}^{\prime 2}-\omega_{d}^{2}}\right]
$$

Where, $y(x, t)$ is the response of deck at position of $x$ and time of $t$ (counted from entrance of the first load) which using $f_{u}(t)$ results in deflection and $f_{a}(t)$ leads to acceleration. $N$ is the number of car-bodies, $p$ is axle load, $L$ is span length of the bridge, $E I$ is the flexural rigidity, $H(t)$ is the Heaviside function, $\omega_{i}$ is the natural frequency of the $i$ th mode of vibration, $\omega_{d}$ is the damped fundamental natural frequency of the bridge, $\omega=\pi v / L$ is the excitation frequency, $\omega_{i}^{\prime}=\sqrt{\omega_{i}^{2}-\omega_{d}^{2}}, \Omega_{i}=\sqrt{\omega_{i}^{\prime 2}+\omega_{d}^{2}}$ and $D=$ $\sqrt{\left(\Omega_{i}^{2}-i^{2} \omega^{2}\right)^{2}+\left(2 i \omega \omega_{d}\right)^{2}}$. It is worthwhile noting that bridge deflection can be calculated accurately by only considering the fundamental mode of vibration; however, acceleration is sensitive to the considered number of modes. Therefore, CEN (2005) recommended to calculate deck acceleration for frequencies up to $\max \left(30 \mathrm{~Hz}, 1.5 f_{1}, f_{3}\right)$, where $f_{1}$ and $f_{3}$ are the frequency of the first and third modes of vibration, respectively.

This approach neglects the contribution of track, rail irregularities and also Train-TrackBridge Interaction (TTBI) effects. It was previously discussed by Jin et al. (2018) that the track structure transforms concentrated axle loads at the rail level into distributed loads (previously considered as either triangular or rectangular loads) at the deck level. This contribution can significantly reduce the dynamic responses, especially for bridges with shorter spans. This is implicitly implemented here by a fitted polynomial equation to the proposed graphical reduction factor in CEN (2003). The adopted reduction factor is presented in Eq.(11).

$R(\lambda)= \begin{cases}0.14 & \lambda \leq 1.5 \\ \min \left[-1.1 e^{-4} \lambda^{4}+5.3 e^{-3} \lambda^{3}\right. & \\ -8.3 e^{-2} \lambda^{2}+0.57 \lambda-0.54 & \lambda>1.5 \\ , 1.0] & (11)\end{cases}$ 
Where, $\lambda$ is the wavelength defined as ratio of the train speed to the fundamental frequency of the bridge.

On the contrary, any imperfection or unevenness in the rail geometry has an impact on the structure. It is obvious that such influences increase the dynamic responses compared to smooth rails, as considered by Frýba (2001). Therefore, CEN (2003) proposed an amplification factor as Eq.(12) for rails with good quality (which is supposed to be the case for high-speed lines). It is worth noting that the codified amplification factor for deflections and accelerations is identical; however, this proposal is questioned by several researchers such as Salcher et al. (2019). Based on their research, Eq.(12) seems to be acceptable for deflections, while it is significantly underestimated for accelerations. Therefore, Eq.(12) is used to amplify the calculated deflections, while the amplification factor corresponding to the accelerations $\left(R I A_{a}\right)$ is considered as a random variable based on the recommendations of Salcher et al. (2019).

$\varphi^{\prime \prime}=\frac{\alpha}{200}\left[56 e^{-(L / 10)^{2}}+50\left(\frac{L f_{1}}{80}-1\right) e^{-(L / 20)^{2}}\right]$

Where, $\alpha=\min (v / 22,1), L$ is the bridge span length and $f_{1}$ is the fundamental frequency of the bridge.

Then, the beneficial contribution of TTBI is considered by adopting the proposed approach of equivalent additional damping $(\Delta \xi)$ in Yau et al. (2019). They have shown that considering the equivalent additional damping in moving load problems can lead to approximately identical responses compared to numerical models with TTBI. The corresponding expression is as presented in Eq.(13).

$$
\Delta \xi=\mu r\left|\frac{r+2 \xi_{v} j}{\left(1-r^{2}\right)-2 \xi_{v} r j}\right|
$$

Where, $\mu$ is the train to bridge modal mass ratio, $r$ is their fundamental frequency ratio in percentage, $\xi_{v}$ is the modal damping of the coach and $j$ is the imaginary unit.

\section{Basic Random Variables}

The considered basic random variables are divided into four categories, those related to the bridge (moment inertia of the deck, mass per length, modulus of elasticity, and material damping), the rail and track (rail irregularity), the train (axle load, bogie distance, carbody length, fundamental frequency and its corresponding damping ratio of the suspension system, and modal mass), and the capacities (vertical acceleration related to the ballast instability and deflection threshold corresponding to passenger comfort).

Despite all the progresses in introducing more accurate computational models, there will always be an error in the predictions with respect to reality. Therefore, the predictions of the numerical model are multiplied by a model uncertainty variable (denoted here as $\chi_{M}$ ). It should be noted that the assigned probability distribution for model uncertainty is based on engineering judgement of the authors. In summary, the associated probability distribution functions for each random variable and the corresponding parameters are given in Tables 1-2. The potential reader interested in the details of such assignments is referred to Allahvirdizadeh et al. (2021).

It should be noted that moment of inertia and mass per length are both a function of cross-sectional dimensions; which makes them interdependent. Therefore, a Student's t-copula probability density function with a linear correlation parameter of $\rho=0.92$ and degree of freedom parameter of $\nu=9.9$ was used by Allahvirdizadeh et al. (2021) to model their dependency. The scatter of the generated sample for the cases where mass per length and moment of inertia are considered independent or dependent are compared in Fig.2.

To the authors' knowledge, there is a lack of information (either measurements or numerical calculations) regarding the deflection threshold of high-speed railway bridges. Performing such work is beyond the scope of the current article; therefore, it was decided to tie this variable to the values given in $\mathrm{CEN}$ (2005). It was not possible to justify a theoretical probability distribution according to the approaches discussed in Allahvirdizadeh et al. (2021). Therefore, a uniform distribution is considered here.

$$
\xi_{b}(L)= \begin{cases}1.5+0.07(20-L) & L \leq 20 \mathrm{~m} \\ 1.5 & L>20 \mathrm{~m}\end{cases}
$$

\section{Training Surrogate Models}

To train the best performing surrogate models, basis-adaptive PCE with a maximum polynomial degree of 10 and a hyperbolic truncation scheme (q-norm) varying in the range of $[0.5-1.0]$ is used. It should be noted that all analyses performed in this study are carried out using the UQLAB toolbox developed by Marelli and Sudret (2014).

Such surrogate models are prone to the problem of over-fitting; which is addressed here by accounting for the leave-one-out cross 
Table 1. Considered basic random variables.

\begin{tabular}{|c|c|c|c|}
\hline Variable & Dist. ${ }^{*}$ & $\begin{array}{l}\text { Mean } \\
\text { Min. } \\
\text { Scale }\end{array}$ & $\begin{array}{l}\text { Std } \\
\text { Max. } \\
\text { Shape }\end{array}$ \\
\hline$I\left(\mathrm{~m}^{4}\right)^{* *}$ & $\mathrm{LN}$ & $\mu_{I}$ & $\sigma_{I}$ \\
\hline$m(\mathrm{~kg} / \mathrm{m})^{\dagger}$ & $\mathrm{LN}$ & $\mu_{m}$ & $\sigma_{m}$ \\
\hline$E(\mathrm{GPa})$ & $\mathrm{N}$ & 29.7 & 3.56 \\
\hline$\xi_{b}(\%)^{\dagger \dagger}$ & $\mathrm{LN}$ & Eq.(14) & 0.30 \\
\hline$p(\mathrm{kN})^{\ddagger}$ & $\mathrm{W}$ & 194.93 & 9.14 \\
\hline$d_{B A}(\mathrm{~m})$ & $\mathrm{U}$ & 2.0 & 3.5 \\
\hline$D(\mathrm{~m})$ & $\mathrm{U}$ & 17.0 & 28.0 \\
\hline$f_{v}(\mathrm{~Hz})$ & $\mathrm{W}$ & 1.04 & 3.07 \\
\hline$m_{v}(\mathrm{~kg})$ & G & $2.2 \mathrm{e} 3$ & 8.6 \\
\hline$\xi_{v}(\%)$ & $\mathrm{W}$ & 16.4 & 1.59 \\
\hline$R I A_{a}(-)$ & $\mathrm{LN}$ & $A f+B$ & $A^{\prime} f+B^{\prime}$ \\
\hline$\chi_{M}(-)$ & $\mathrm{N}$ & 0.0 & 0.10 \\
\hline$a_{l i m}\left(\mathrm{~m} / \mathrm{s}^{2}\right)$ & $\mathrm{N}$ & $0.8 \mathrm{~g}$ & $0.1 \mathrm{~g}$ \\
\hline$(L / \delta)_{\lim }(-)^{\ddagger \ddagger}$ & $\mathrm{U}$ & $(L / \delta)_{l o}$ & $(L / \delta)_{u p}$ \\
\hline
\end{tabular}

Note: * N, LN, U, W and G stand for Normal, Log-Normal, Uniform, Weibull and Gamma distributions, respectively.

** The distribution is truncated for values less than $0.083 \mathrm{~m}^{4}, 0.58 \mathrm{~m}^{4}$ and $1.4 \mathrm{~m}^{4}$ for bridges with 10 $\mathrm{m}, 20 \mathrm{~m}$ and $30 \mathrm{~m}$ span lengths, respectively.

$\dagger$ Based on recommendations of Museros et al. (2020), the distribution should be truncated for values less than $400 L+4900 \mathrm{~kg} / \mathrm{m}$.

$\dagger^{\dagger}$ Parameters of the distribution are reported in physical space.

$\ddagger$ The distribution is truncated for values less than $120 \mathrm{kN}$.

$\ddagger \ddagger$ The distribution is truncated for values less than $0.6 \mathrm{~g}$ and greater than $1.0 \mathrm{~g}$.

validation error. Moreover, the accuracy of the model for the prediction objectives is verified by limiting this error to 0.001 .

An example of trained surrogate models for predicting the maximum vertical acceleration for bridges with a span length of $20 \mathrm{~m}$ is
Table 2. Parameters of basic random variables dependent on span length.

\begin{tabular}{|c|c|c|c|}
\hline \multirow{2}{*}{ 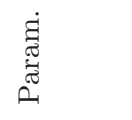 } & \multicolumn{3}{|c|}{$L(\mathrm{~m})$} \\
\hline & 10 & 20 & 30 \\
\hline$\mu_{I}$ & -1.12 & 0.097 & 0.720 \\
\hline$\sigma_{I}$ & 0.460 & 0.340 & 0.400 \\
\hline$\mu_{m}$ & 9.290 & 9.720 & 9.920 \\
\hline$\sigma_{m}$ & 0.320 & 0.260 & 0.230 \\
\hline$A$ & 0.005 & 0.018 & 0.040 \\
\hline$B$ & -0.011 & 0.009 & 0.120 \\
\hline$A^{\prime}$ & 0.007 & 0.014 & 0.025 \\
\hline$B^{\prime}$ & -0.001 & 0.046 & 0.070 \\
\hline$(L / \delta)_{l o}$ & 700 & 780 & 935 \\
\hline$(L / \delta)_{u p}$ & 1050 & 1050 & 1110 \\
\hline
\end{tabular}
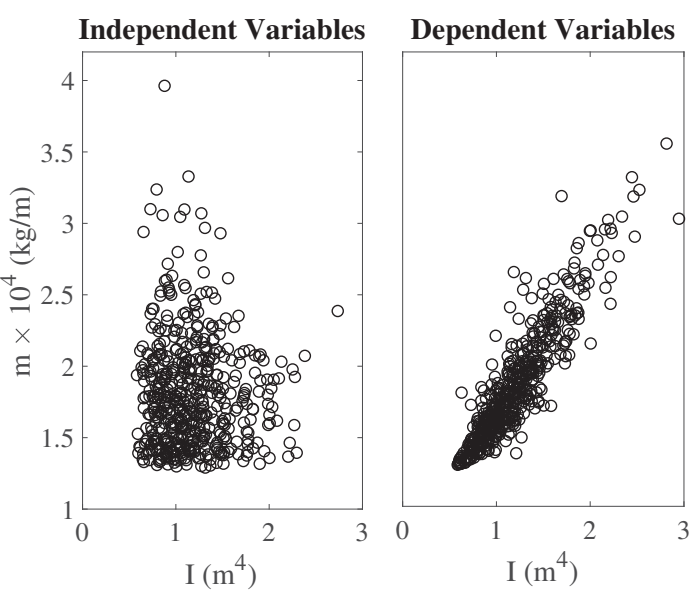

Fig. 2. Comparison between dependency status of mass per length and moment of inertia, an example for bridges with span length of $20 \mathrm{~m}$

presented in Fig.3.

\section{Performance of Subset Simulation}

As discussed, the performance of subset simulation is a function of $p_{0}$ and $N_{s}$. Therefore, 


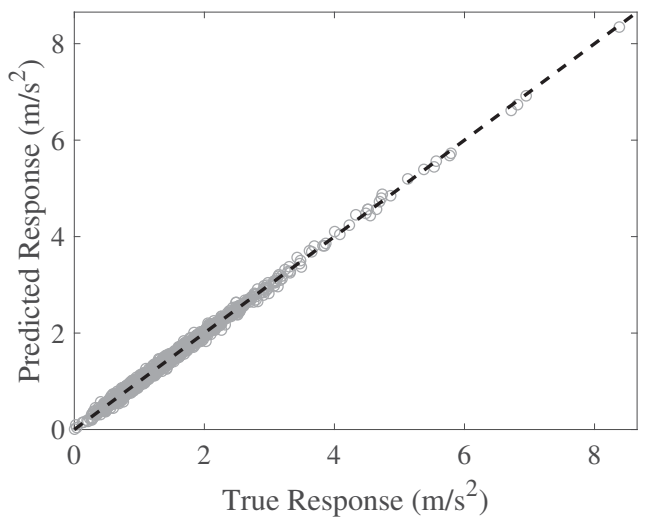

Fig. 3. Trained PCE surrogate model to predict maximum vertical acceleration of bridges with span length of $20 \mathrm{~m}$

three and five different values for $p_{0}$ and $N_{s}$ are considered respectively, to investigate the sensitivity of the results to each of these values and also to find the best configuration. It should be noted that in the cases where $p_{0}$ is varied, the value of $N_{s}$ is fixed at 1000 . Similarly, in the cases of investigating the influence of $N_{s}$, the value of 0.1 is assigned to $p_{0}$.

Examples of the aforementioned sensitivity analyses for the running safety assessment of bridges with a span length of $20 \mathrm{~m}$ are shown in Figs.4-5. When considering the resulting coefficients of variation from each case, it was found that the results are more sensitive to the number of samples. Furthermore, considering $N_{s}=4000$ can lead to sufficiently stable results for all operational train speeds. Therefore, further subset simulations were performed with $p_{0}=0.1$ and $N_{s}=4000$.

It is worth noting that, as expected, a large coefficient of variation was obtained for the calculated violation probabilities at lower operating train speeds. This is due to the much smaller probabilities of failure at these speeds. Therefore, the performance of the subset simulation can be improved by considering a larger sample size for lower speeds; however, this approach is neglected here.

\section{Surrogate Assisted Monte-Carlo versus Subset Simulation}

In this section, the performance of the Latin Hypercube-sampling-based Monte-Carlo approach supported by trained PCE surrogate models is compared with subset simulation. For this purpose, the results of the crude

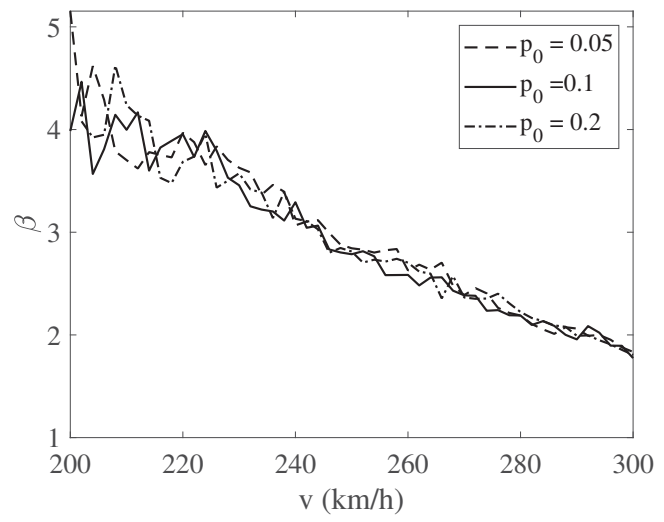

Fig. 4. Sensitivity of subset simulation for $p_{0}$ in evaluation of running safety for bridges with span length of $20 \mathrm{~m}$

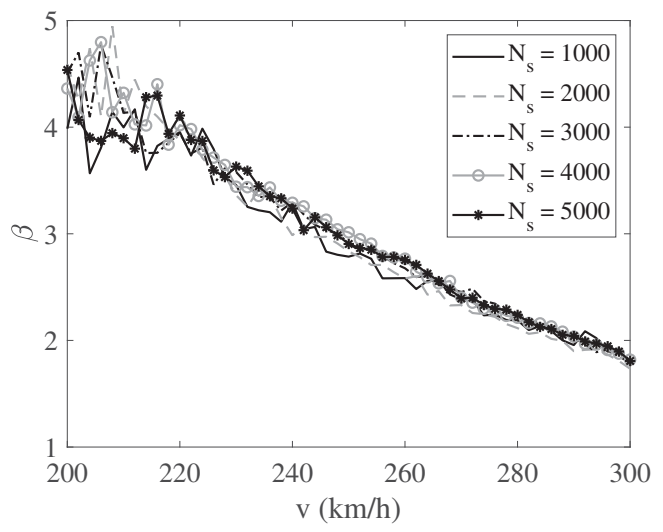

Fig. 5. Sensitivity of subset simulation for $N_{s}$ in evaluation of running safety for bridges with span length of $20 \mathrm{~m}$

Monte-Carlo method with a sample size of $10^{6}$ for bridges with $20 \mathrm{~m}$ span length and at an operational train speed of $240 \mathrm{~km} / \mathrm{h}$ are considered as benchmark solution. These comparisons are presented in Tables 3-4. It should be emphasized that the elapsed time values are the ratio of the computation time of each method to that of the benchmark solution. Moreover, the spent time on finding the optimum polynomial degree and truncation scheme for PCE surrogate models is not included here, i.e. the reported time is based on the best working configuration.

As can be seen, both approaches can sig- 
nificantly reduce the computation time without sacrificing accuracy; however, the resulted safety index by subset simulation is closer to the true value (at least in the considered benchmark situation). Therefore, the targeted stochastic comparison is performed using the subset simulation method.

Table 3. Comparison between different reliability assessment methods for running safety limit stat, at operating train speed of $240 \mathrm{~km} / \mathrm{h}$.

\begin{tabular}{llll}
\hline Method & $\beta$ & c.o.v & $\begin{array}{l}\text { Elapsed } \\
\text { Time }\end{array}$ \\
\hline MC & 3.428 & 0.057 & 1 \\
$\begin{array}{l}\text { Subset } \\
\text { Simulation }\end{array}$ & 3.236 & 0.165 & 0.016 \\
PCE+MC & 2.967 & 0.026 & 0.011 \\
\hline
\end{tabular}

Table 4. Comparison between different reliability assessment methods for passenger comfort limit state at operating train speed of $240 \mathrm{~km} / \mathrm{h}$.

\begin{tabular}{llll}
\hline Method & $\beta$ & c.o.v & $\begin{array}{l}\text { Elapsed } \\
\text { Time }\end{array}$ \\
\hline MC & 3.916 & 0.0057 & 1 \\
$\begin{array}{l}\text { Subset } \\
\text { Simulation }\end{array}$ & 3.874 & 0.161 & 0.017 \\
PCE+MC & 3.620 & 0.082 & 0.017 \\
\hline
\end{tabular}

7. Stochastic Comparison Between

Running Safety and Passenger

Comfort

Finally, the violation probability of running safety and passenger comfort limit states are compared at desired operating train speeds and for considered bridges in Fig.6. As can be seen, in most cases the running safety criterion would dominate; however, the difference decreases as the bridge span length increases. Moreover, it seems that if the reliability-based design optimization is aimed to be performed for bridges with larger spans $(L>20 \mathrm{~m})$ and operating train speeds lower than 240 $\mathrm{km} / \mathrm{h}$, the multi-objective optimization should be conducted.
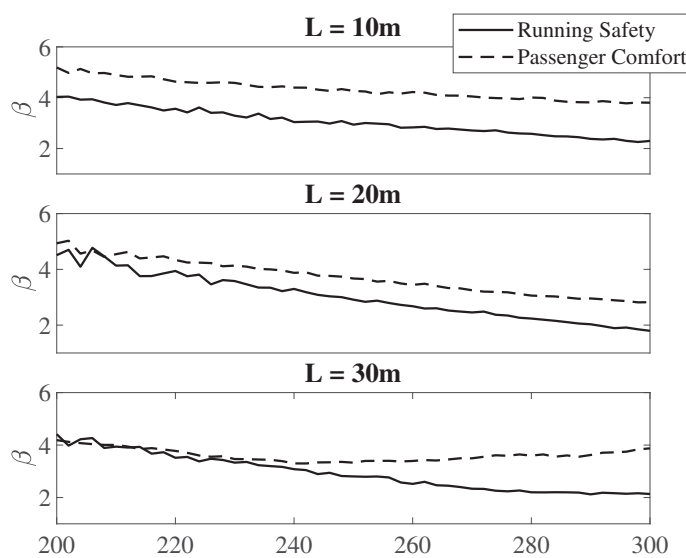

Fig. 6. Stochastic comparison between running safety and passenger comfort

\section{Conclusions}

In this study, a stochastic comparison between two design criteria of high-speed railway bridges, i.e. running safety and passenger comfort for different bridge span lengths and operating train speeds is conducted. The surrogate-based Monte-Carlo method and subset simulation are used for this objective. Both approaches can speed up the reliability assessment procedure; therefore, their performance is compared with the benchmark method, i.e crude Monte-Carlo. The comparison includes both the computation time and the error in the estimated violation probability (safety index). It was found that both methods require a computation time of about less than $2 \%$ of the crude Monte-Carlo; however, the computed safety index by the subset simulation was closer to that of the benchmark solution. Therefore, the subset simulation method is adopted to evaluate the safety index corresponding to running safety and passenger comfort. Based on the obtained results, running safety would control the design particularly for shorter span bridges; however, multi-objective design optimization should be performed for bridges larger than $20 \mathrm{~m}$ and operating train speeds lower than $240 \mathrm{~km} / \mathrm{h}$. 


\section{Acknowledgement}

This project has received funding from the Shift2Rail Joint Undertaking under the European Unions Horizon 2020 research and innovation program under grant agreement No. 826255.

\section{References}

Allahvirdizadeh, R., A. Andersson, and R. Karoumi (2020). Reliability assessment of the dynamic behavior of high-speed railway bridges using first order reliability method. In Eurodyn 2020, 11th International Conference on Structural Dynamics, Volume 2, pp. 3438-3450.

Allahvirdizadeh, $\quad$ R., $\quad$ A. Andersson, and R. Karoumi (2021). Minimum design requirements of high-speed railway bridges using reliability-based optimization. Unpublished Manuscript.

Arvidsson, T. (2018). Train-track-bridge interaction for the analysis of railway bridges and train running safety. Ph. D. thesis, KTH Royal Institute of Technology.

Au, S.-K. and J. L. Beck (2001). Estimation of small failure probabilities in high dimensions by subset simulation. Probabilistic engineering mechanics 16(4), 263-277.

CEN (2003). EN 1991-2, Eurocode 1: Actions on structures - part 2: Traffic loads on bridges. European Committee for Standardization.

CEN (2005). EN 1990/A1, basis of structural design annexe A2. European Committee for Standardization.

Frýba, L. (2001). A rough assessment of railway bridges for high speed trains. Engineering Structures 23 (5), 548-556.

Jin, Z., B. Huang, J. Ren, and S. Pei (2018). Reduction of vehicle-induced vibration of railway bridges due to distribution of axle loads through track. Shock and Vibration 2018.

Marelli, S. and B. Sudret (2014). Uqlab: A framework for uncertainty quantification in matlab. In Vulnerability, uncertainty, and risk: quantification, mitigation, and management, pp. 2554-2563.

Museros, P., A. Andersson, V. Martí, and R. Karoumi (2020). Dynamic behaviour of bridges under critical articulated trains: Signature and bogie factor applied to the review of some regulations included in en 1991-2. Proceedings of the Institution of Mechanical Engineers, Part F: Journal of Rail and Rapid Transit 235(5), 655-675.

Salcher, P., C. Adam, and A. Kuisle (2019). A stochastic view on the effect of random rail ir- regularities on railway bridge vibrations. Structure and Infrastructure Engineering 15(12), 1649-1664.

Sudret, B. (2008). Global sensitivity analysis using polynomial chaos expansions. Reliability engineering \& system safety 93(7), 964-979.

Xiu, D. and G. E. Karniadakis (2002). The wiener-askey polynomial chaos for stochastic differential equations. SIAM journal on scientific computing 24(2), 619-644.

Yau, J., M. Martínez-Rodrigo, and A. Doménech (2019). An equivalent additional damping approach to assess vehicle-bridge interaction for train-induced vibration of short-span railway bridges. Engineering Structures 188, 469-479.

Zacher, M. and M. Baeßler (2008). Dynamic behaviour of ballast on railway bridges. In Dynamics of High-Speed Railway Bridges. Selected and Revised Papers from the Advanced Course on 'Dynamics of High-Speed Railway Bridges', Porto, Portugal, 20-23 September 2005. CRC Press. 